\title{
Implicaciones de los choques de prima de riesgo en una economía pequeña y abierta
}

José Mauricio Gil-León y Andrés Felipe Suárez-Cante 
Lecturas de Economía, 92 (enero-junio 2020), pp. 133-172

José Mauricio Gil-León y Andrés Felipe Suárez-Cante

\title{
Implicaciones de los choques de prima de riesgo en una economía pequeña y abierta
}

Resumen: En este articulo se pretende identificar el canal de transmisión de los choques de prima de riesgo en las diferentes variables macroeconómicas. Por tanto, se formula un modelo DSGE para una economía pequeña y abierta, que considera el comportamiento de los hogares, las decisiones de inversión de parte de las empresas, la función de reacción del banco central y la dinámica de las diferentes variables externas, tales como la variación de los activos externos netos del país y el comercio exterior. El modelo se calibra con datos de la economía colombiana del periodo 2005-2017. En los resultados de la simulación se identifica la persistencia del shock de prima de riesgo en las variables endógenas que componen al modelo, y en especial se observan efectos sobresalientes del choque sobre el tipo de cambio, la tasa de interés y la inflación. Se concluye que el tamaño de la elasticidad de la prima de riesgo al endeudamiento externo domina la fuerza con la que inciden los shocks en la economía.

Palabras clave: modelo DSGE; prima de riesgo; tasa de interés; tasa de cambio.

Clasificación JEL: C63, C68, E47, F37, F41.

\section{Implications of risk premium shocks in a small and open economy}

\begin{abstract}
This paper aims to identify the transmission channel of risk premium shocks in different macroeconomic variables. Therefore, a DSGE model for a small and open economy is formulated, which considers the behavior of households, investment decisions by companies, the reaction function of the central bank and the dynamics of the different external variables, such such as the variation of the country's net foreign assets and foreign trade. The model is calibrated with data from the Colombian economy of the 2005-2017 period. In the simulation results, the persistence of the risk. premium shock in the endogenous variables that make up the model is identified, and in particular, outstanding effects of the shock on the exchange rate, the interest rate and inflation are observed. It is concluded that the size of the elasticity of the risk premium to external indebtedness dominates the force with which the shocks affect the economy.
\end{abstract}

Keywords: DSGE models; risk premium; interest rate; exchange rate.

JEL Classification: C63, C68, E47, F37, F41.

\section{Implications des chocs de primes de risque dans une petite économie ouverte}

Résumé: Cet article vise à identifier le canal de transmission des chocs de primes de risque dans différentes variables macroéconomiques. Pour ce faire, un modèle DSGE est formulé pour une petite économie ouverte, prenant en compte le comportement des ménages, les décisions d'investissement des entreprises, la fonction de réaction de la banque centrale et la dynamique des différentes variables externes, telles que la variation des actifs extérieurs nets du pays et le commerce extérieur. Le modèle est ensuite calibré avec les données de l'économie colombienne pour la période 2005-2017. Les résultats de la simulation montrent la persistance du choc de prime de risque dans les variables endogènes du modèle. En particulier, nous montrons les effets remarquables du choc sur le taux de change, sur le taux d'intérêt et sur l'inflation. Nous concluons que l'ampleur de l'élasticité de la prime de risque à l'endettement extérieur domine la force avec laquelle les chocs touchent l'économie.

Mots clés: modèle DSGE; prime de risque; taux d'intérêt; taux de change.

Classification JEL: C63, C68, E47, F37, F41. 


\title{
Implicaciones de los choques de prima de riesgo en una economía pequeña y abierta
}

\author{
José Mauricio Gil-León (10 ${ }^{\mathrm{a}}$ y Andrés Felipe Suárez-Cante $\mathbb{1}^{\mathrm{b}}$ \\ -Introducción. -I. El modelo. -II. Calibración del modelo. -III. Análisis de resultados. \\ -Conclusiones. -Referencias. -Anexos.
}

doi: 10.17533/udea.le.n92a05

Primera versión recibida el 17 de noviembre de 2018; versión final aceptada el 9 de septiembre de 2019

\section{Introducción}

El análisis de la prima de riesgo y su modelación en una forma estructural no está vinculado dentro de un marco específico de teoría económica y, por ende, su incidencia en la economía es poco predecible (Iranzo, 2008). La estimación de la prima de riesgo no tiene una única metodología, y por ello su determinación depende de la calificación del riesgo, el cual, a su vez, depende del riesgo político, el riesgo económico y los riesgos naturales. Sin embargo, varias entidades financieras, y en especial las del sector bancario, se apoyan en diferentes instrumentos analíticos, estadísticos y econométricos para determinar el riesgo país (Iranzo, 2008).

Los efectos de la prima de riesgo en la economía se han analizado explícitamente en la literatura a través de modelos econométricos (Eraker \& Wang, 2015). El efecto más estudiado ha sido la incidencia de las variaciones de la prima de riesgo sobre el tipo de interés y la inflación. En el trabajo

a José Mauricio Gil-León: profesor de planta, Escuela de Economía, Universidad Pedagógica y Tecnológica de Colombia, Colombia. Economista y magíster en Economía. Dirección postal: avenida central del norte, Tunja. Dirección electrónica: josemauricio.gil@uptc.edu.co https://orcid.org/0000-0002-5653-5245

b Andrés Felipe Suárę-Cante: economista, matemático y magíster en Economía de la Universidad Pedagógica y Tecnológica de Colombia, Colombia. Dirección postal: Avenida central del norte, Tunja. Dirección electrónica: andres.suarez@uptc.edu.co https://orcid.org/0000-0001-9617-7879 
Gil-León y Suárez-Cante: Implicaciones de los choques de prima de riesgo en una economía...

de Pienika (2014), dichas variaciones provocan una disminución tanto de la inflación como del tipo de cambio. Sin embargo, Melo y Riascos (2004) encontraron que un choque en la prima de riego genera un aumento del tipo de interés y una disminución inicial de la inflación con posterior tendencia creciente, lo cual es confirmado en Gelain (2010) con la relación positiva entre el choque de prima de riesgo, el tipo de interés y la inflación. Esta tendencia en el análisis, junto a la dificultad para identificar el mecanismo de transmisión de los choques de riesgo país en un modelo estructural lleva a preguntarse: ¿Cuáles son los efectos de los cambios en la prima de riesgo de Colombia sobre las diferentes variables macroeconómicas?

El problema de investigación hace necesario utilizar un modelo de equilibrio general dinámico y estocástico (DSGE: dynamic stochastic general equilibrium, en inglés) para analizar las fuerzas económicas que hay detrás de los movimientos en las primas de riesgo de los bonos y su influencia en los agregados macroeconómicos. La construcción del modelo se justifica en la poca atención que se le ha dado en la literatura a la prima de riesgo y a su papel como determinante del comportamiento de diversas variables macroeconómicas. En particular, es necesario identificar el canal de transmisión en las economías emergentes como la colombiana.

La inclusión de la prima de riesgo en modelos DSGE se ha realizado en trabajos como el de Rudebusch y Swanson (2008), en donde se define una economía de producción y se explica cómo las primas de riesgo tienen incidencia directa sobre variables financieras como el patrimonio, los bonos reales, los bonos nominales y los tipos de cambio. Por otro lado, Graeve, Dossche, Emiris, Sneessens y Wouters (2010) analizan de manera explícita modelos financieros en los cuales la prima de riesgo y el precio de los riesgos observados durante la contratación de trabajadores tienen un papel importante en la estabilidad financiera de la economía. En esta línea de trabajo, Papantonis (2016) determina las implicaciones de capturar la volatilidad de la prima de riesgo a través de un modelo autorregresivo de heterocedasticidad condicional generalizado (GARCH) y de incluirlo en un DSGE. Con el mismo método, Posch (2009) demuestra cómo las no linealidades y no normalidades son importantes para generar predicciones: el autor observó empíricamente hechos estilizados de la prima de riesgo y confirmó que estas características 
claves pueden explicar el puz₹le de prima de acciones y el comportamiento variable en el tiempo de la prima de riesgo. Para esto empleó soluciones explícitas de modelos DSGE, donde observó que las no linealidades pueden generar variaciones en las primas de riesgo que son observables. Los DSGE formulados en Colombia, tales como los de González, Mahadeva, Prada y Rodríguez (2011), González, et al. (2014), y Suescún y Steiner (2017), han involucrado la dinámica de la prima de riesgo al interior de los modelos, pero no identifican la trayectoria del shock sobre las demás variables.

\section{El modelo}

La estructura del modelo que aquí se emplea se basa en los trabajos de Alendal (2008) y Vallejos y Navia (2016), los cuales comparten elementos fundamentales. Dicho modelo asume una economía pequeña y abierta con mercados de bienes no competitivos y rigideces nominales. Lo último implica que existen rigideces salariales, considerando el supuesto de que los hogares delegan en un sindicato el poder de negociar salarios cada periodo (Galí, 2008). Se modela el comportamiento de los hogares, las empresas, el sector externo y el banco central. Así mismo, el shock en el riesgo país se define como una función de la capacidad de las empresas y hogares para obtener financiamiento externo (nivel relativo de activos externos netos), por lo que las condiciones de la cuenta corriente determinan la dinámica de la prima de riesgo. No se incluyen directamente las decisiones del gobierno, pero en la dinámica de la cuenta corriente el ahorro del gobierno, del sector privado y el externo es relevante, por lo que un mayor déficit fiscal implica menor ahorro interno y una mayor necesidad de financiamiento externo (déficits gemelos).

Las ecuaciones provienen de la solución de problemas de optimización de agentes y de formulaciones ad hoc, lo cual define al modelo analizado como un DSGE semiestructural. Además, se incluye formación de hábitos en el consumo, los cuales son incluidos para replicar la persistencia empírica de los datos. El listado de variables y parámetros se define en la Tabla 1. 
Gil-León y Suárez-Cante: Implicaciones de los choques de prima de riesgo en una economía...

Tabla 1. Lista de simbolos y convenciones del modelo

\begin{tabular}{|c|c|}
\hline Variable Parámetro & Descripción \\
\hline \multicolumn{2}{|r|}{ Canasta de consumo } \\
\hline$C_{d, t}$ & Canasta de consumo del bien producido domésticamente \\
\hline$C_{i m, t}$ & Canasta de consumo del bien importado \\
\hline$e_{d}$ & $\begin{array}{l}\text { Elasticidad de sustitución entre variedades de bienes y servicios } \\
\text { nacionales }\end{array}$ \\
\hline$e_{i m}$ & $\begin{array}{l}\text { Elasticidad de sustitución entre variedades de bienes y servicios } \\
\text { extranjeros }\end{array}$ \\
\hline$e$ & $\begin{array}{l}\text { Elasticidad de sustitución entre bienes de consumo nacionales e } \\
\text { importados }\end{array}$ \\
\hline$\alpha$ & $\begin{array}{l}\text { Paramento indicativo del grado de apertura de la economía nacional } \\
\text { al resto del mundo }\end{array}$ \\
\hline$G H_{t}$ & Gasto total de los hogares \\
\hline$P_{d, t}$ & Nivel de precios de bienes domésticos \\
\hline$P_{i m, t}$ & Precio de bienes importados \\
\hline$P_{t}^{*}$ & Índice de precios mundiales \\
\hline$\epsilon_{t}$ & Tipo de cambio nominal \\
\hline$P_{T, t}$ & Precios agregados "nivel general de precios" \\
\hline \multicolumn{2}{|r|}{ Inflación y tipo de cambio real } \\
\hline$\pi_{t}$ & Tasa de inflación \\
\hline$\pi_{d, t}$ & Tasa de inflación doméstica \\
\hline$\pi_{t}^{*}$ & Tasa de inflación importada \\
\hline$S_{t}$ & Términos de intercambio \\
\hline$T C R_{t}$ & Tipo de cambio real \\
\hline \multicolumn{2}{|r|}{ Banco central } \\
\hline$i_{t}$ & Tasa de interés nominal \\
\hline $\bar{Y}$ & PIB potencial \\
\hline $\bar{r}$ & Tasa real esperada \\
\hline $\bar{\pi}$ & Inflación esperada \\
\hline
\end{tabular}

Continúa 
Tabla 1. Continuación

\begin{tabular}{|c|c|}
\hline Variable Parámetro & Descripción \\
\hline$f_{i}$ & Peso de la brecha del interés pasado en la relación de la tasa de política \\
\hline$f_{\pi}$ & Peso de la brecha de la inflación en la reacción de la tasa de política \\
\hline$f_{Y}$ & $\begin{array}{l}\text { Peso de la brecha del producto en la reacción de la tasa de interés de } \\
\text { política }\end{array}$ \\
\hline \multicolumn{2}{|r|}{ Ecuación de la paridad de los activos } \\
\hline$B_{t}$ & Nivel de activos en forma de bonos \\
\hline$Y_{t}$ & Nivel de producción agregado de la economía \\
\hline \multicolumn{2}{|r|}{ Paridad de tasas de interés y prima de riesgo } \\
\hline$P R_{t}$ & Prima de riesgo \\
\hline$\mu$ & Elasticidad de la prima de riesgo \\
\hline $\bar{B}$ & $\begin{array}{l}\text { Nivel de activos agregado del exterior en forma de bonos en estado } \\
\text { estacionario }\end{array}$ \\
\hline$\varphi_{t}$ & Shock estocástico en prima de riesgo \\
\hline$\hat{i}_{t}^{*}$ & Tasa de interés nominal del extranjero \\
\hline \multicolumn{2}{|r|}{ Los hogares } \\
\hline$\eta$ & Ajuste de hábito de consumo \\
\hline$\ni$ & Inverso de la elasticidad de sustitución intertemporal \\
\hline$\gamma$ & Elasticidad, oferta de trabajo \\
\hline$\beta$ & Factor de descuento intertemporal \\
\hline$L_{t}$ & Cantidad de horas trabajadas \\
\hline$w_{t}$ & Salario real \\
\hline$W_{t}$ & Salario nominal \\
\hline$G H_{t}$ & Gasto de los hogares \\
\hline \multicolumn{2}{|r|}{ Inversión y capital } \\
\hline$I N_{t}$ & Función de inversión \\
\hline$i_{T, t}$ & Función de tasa de interés entre el interés nacional y extranjero \\
\hline$\vartheta$ & Elasticidad de la inversión respecto al interés total \\
\hline$K_{t}$ & Función de capital \\
\hline
\end{tabular}

Continúa 
Gil-León y Suárez-Cante: Implicaciones de los choques de prima de riesgo en una economía...

Tabla 1. Continuación

\begin{tabular}{cl}
\hline Variable Parámetro & \multicolumn{1}{c}{ Descripción } \\
\hline$\sigma$ & Tasa de depreciación física del capital \\
\hline$\delta$ & Elasticidad del nivel de producción respecto al capital \\
$L_{t}$ & Número total de horas dedicadas a trabajar \\
$P M L_{t}$ & Productividad marginal del trabajo \\
$A_{t}$ & Estado de la tecnología o productividad total de los factores \\
$C G_{t}$ & Costo marginal \\
$\rho$ & Parámetro asociado a la rigidez de precios \\
\hline$I M_{t}$ & Sector externo \\
$X_{t}$ & Exportaciones \\
$X M_{t}$ & Balanza comercial \\
$\phi$ & Elasticidad de las exportaciones frente a la economía externa \\
$e^{*}$ & Elasticidad de la sustitución de bienes del extranjero \\
\hline$\tau$ & Condición de equilibrio \\
\hline$\chi_{r}$ & Parámetro de ajuste a la inversión \\
\hline & Shock \\
\hline
\end{tabular}

Fuente: elaboración propia.

\section{A. Hogares}

Se modela el comportamiento de los hogares considerando rigideces salariales y una oferta de mano de obra diferenciada. Las decisiones de consumo y ahorro se toman en mercados completos, y los hogares toman los precios de los bienes como dados, asegurándolos contra los riesgos intrínsecos y salariales (Woodford, 2003). Esto hace que los hogares sean esencialmente iguales en equilibrio, lo cual establece que el hogar es 
homogéneo y representativo. Esto posibilita prescindir de las complejidades que se derivan de la heterogeneidad del hogar.

Además, se puede establecer que un hogar promedio tiene riqueza financiera en forma de activos nominales denominados en moneda nacional que son emitidos por bancos comerciales, empresas privadas y gobierno, y pagan una tasa de interés nominal. Dichos activos consumen un paquete de bienes nacionales e importados, y no pueden asegurar sus ingresos reales contra el efecto de la inflación doméstica, la inflación extranjera ni la evolución del tipo de cambio.

Así, los hogares maximizan una función de utilidad intertemporal para el valor esperado de su utilidad futura sujeto a la restricción presupuestal; para esto, se escoge un nivel de consumo $C_{T, t}$ y de trabajo $L_{t}$, relacionados mediante la siguiente función de utilidad:

$$
U\left(C_{T, t}, L_{t}\right)=\frac{\left(C_{T, t}-\eta C_{T, t-1}\right)^{1-\ni}}{1-\ni}-\frac{L_{t}^{1+\gamma}}{1+\gamma},
$$

donde se agrega $\eta C_{T, t-1}$ para suavizar la curva de consumo, ya que se está incorporando la formación de hábitos para el hogar optimizador (López \& Ramírez, 2011 y Hernández, 2013). Agregando todos los periodos para el nivel de consumo $C_{T, t} \mathrm{y}$ de trabajo $L_{t}$, se tiene que:

$$
\operatorname{máx}_{\left(C_{T, t}, B_{t+1}, L_{t}\right)} E_{t} \sum_{j=1}^{\infty} \beta^{j} U\left(C_{T, t+j}, L_{T, t+j}\right),
$$

sujeto a:

$$
P_{T, t} C_{T, t}=W_{t} L_{t}+\left(1+i_{t}\right) B_{b, t}-B_{b, t+1} .
$$

Las condiciones de primer orden permiten deducir:

$$
\left\{\begin{array}{cccc}
\left(C_{T, t}-\eta C_{T, t-1}\right)^{-\ni} & = & \lambda_{t} P_{T, t} & \\
L_{t}^{\gamma} & = & \lambda_{t} W_{t} & {[A]} \\
\beta E_{t}\left[\left(C_{T, t+1}-\eta C_{T, t}\right)^{-\ni}\right] & = & \lambda_{t+1} P_{T, t+1} & {[C]} \\
\lambda_{t+1}\left(1+i_{t}\right) & = & \lambda_{t} & {[D]}
\end{array}\right.
$$


Gil-León y Suárez-Cante: Implicaciones de los choques de prima de riesgo en una economía...

Dividiendo $[A]$ y $[B]$ se determina el salario real:

$$
\frac{W_{t}}{P_{T, t}}=\frac{L_{t}^{\gamma}}{\left(C_{T, t}-\eta C_{T, t-1}\right)^{-\ni}} .
$$

Realizando una manipulación algebraica y linealizando las expresiones obtenidas por el método de Uhilg se deduce la curva IS dinámica:

$$
\begin{aligned}
\frac{-\ni}{1-\eta}\left(\hat{c}_{T, t-1}-\eta \hat{c}_{T, t-1}\right) & = \\
& \frac{-\ni}{1-\eta} E_{t}\left[\left(\hat{c}_{T, t+1}-\eta \hat{c}_{T, t}\right)\right]+\hat{i}_{t}-E_{t}\left[\widehat{\pi}_{t+1}\right] .
\end{aligned}
$$

La dinámica del consumo está determinada negativamente por la tasa de interés real y positivamente por las expectativas de consumo futuro.

\section{B. Empresas}

Las empresas son las unidades productivas de la economía para un periodo determinado. Estas deciden el nivel de producción $\left(Y_{t}\right)$ a partir de los factores productivos capital y trabajo que tienen que alquilar y contratar. Las empresas tienen una función de producción Cobb-Douglas, donde $A_{t}$ es la productividad total de los factores (PTF), $K_{t}$ es el capital físico utilizado por la empresa y $L_{t}$ es el número de trabajadores. Estos factores productivos se utilizan para producir los bienes finales:

$$
Y_{t}=A_{t} K_{t}^{\delta} L_{t}^{1-\delta}
$$

El problema que resuelven las empresas consiste en la maximización de beneficio:

$$
\operatorname{máx}_{\left(K_{t}, L_{t}\right)} \Pi_{t}=Y_{t}-w_{t} L_{t}-i_{t} K_{t},
$$

cuyas condiciones de primer orden son:

i. $\frac{\partial Y_{t}}{\partial K_{t}}=i_{t}$ 
ii. $\frac{\partial Y_{t}}{\partial L_{t}}=w_{t}$.

De ii se deduce que la productividad marginal del trabajo es igual al salario real: $P M L_{t}=\boldsymbol{w}_{t}$. Obteniendo dicha productividad $P M L_{t}=\frac{\partial Y_{t}}{\partial L_{t}}=$ $(1-\delta) \frac{Y_{t}}{L_{t}}$ y linealizando esta expresión, se tiene que $\widehat{p m l}_{t}=\hat{y}_{t}-\hat{l}_{t}$. Adicional al problema de las empresas y considerando que $w_{t}=\frac{W_{t}}{P_{T}}$, se concluye que $P M L_{t}=\frac{W_{t}}{P_{T}}$. Teniendo en cuenta que del equilibrio de mercado de trabajo se tiene el costo marginal igual al precio ajustado, entonces $C M G_{t}=\frac{P_{T, t}}{P_{d, t}}$; es decir, en términos lineales, $\widehat{c m g}_{t}=\hat{w}_{t}-\widehat{p m l}_{t}-\hat{p}_{d, t}$.

Después de algunas manipulaciones algebraicas se define que:

$$
\begin{aligned}
& \widehat{c m g}_{t}=\frac{(\gamma+\delta)}{(1-\delta)} \hat{y}_{t}-\frac{(\gamma+1) \hat{a}_{t}}{(1-\delta)}-\frac{(\gamma+1) \delta \hat{k}_{t}}{(1-\delta)} \\
& +\frac{\ni}{1-\eta}\left(\hat{c}_{T, t}-\eta \hat{c}_{T, t-1}\right)+\alpha .
\end{aligned}
$$

Como la rigidez de precios origina una inflación doméstica, es esta inflación la que se debe considerar (Galí, 2008). La curva de Phillips es expresada solo en términos de la inflación del hogar, de la siguiente manera $\widehat{\pi}_{d, t}=\beta E_{t}\left[\widehat{\pi}_{d, t+1}\right]+\rho \widehat{c m g}_{t}$; pero combinando con (6) se obtiene la especificación de la curva de Phillips neokeynesiana con formación de hábitos de consumo para una economía abierta:

$$
\begin{aligned}
& \widehat{\pi}_{d, t}=\beta E_{t}\left[\widehat{\pi}_{d, t+1}\right]+ \\
& \rho\left[\frac{(\gamma+\delta)}{(1-\delta)} \hat{y}_{t}-\frac{(\gamma+1)}{(1-\delta)} \hat{a}_{t}-\frac{(\gamma+1) \delta}{(1-\delta)} \hat{k}_{t}+\frac{\ni}{1-\eta}\left(\hat{c}_{T, t}-\eta \hat{c}_{T, t-1}\right)+\alpha \hat{s}_{t}\right] .
\end{aligned}
$$

La inflación doméstica es determinada por las expectativas de esa inflación, la brecha del producto y la inflación importada (el grado de transmisión depende del nivel de apertura que se tenga). En conclusión, la inflación en el periodo $t$ se define como una desagregación entre inflación doméstica e inflación importada: $\widehat{\pi}_{t}=\widehat{\pi}_{d, t}+\alpha \Delta \hat{s}_{t}$. La inflación importada depende positivamente de los términos de intercambio y del grado de apertura de la economía $\alpha$. 
Gil-León y Suárez-Cante: Implicaciones de los choques de prima de riesgo en una economía...

\section{Paridad de tasas de interés y prima de riesgo}

La paridad de los tipos de interés establece que la rentabilidad de los activos con riesgo debe ser igual, independientemente del país donde estén invertidos dichos activos. De esta manera, en la determinación del tipo de interés de un activo de un país considera el tipo de interés externo, la prima de riesgo, el tipo de cambio actual y el tipo de cambio esperado. Por eso se incorpora una función $P R_{t}(\cdot)$, la cual expresa el componente de prima de riesgo de la paridad de las tasas de interés y el efecto sobre la tasa de interés del título de deuda a medida que esta aumenta, y es común para todos los hogares. En otras palabras, la prima de riesgo es el sobrecosto que tiene un emisor de deuda frente a otro emisor de deuda que se considera como referencia y que tiene menor riesgo.

La prima de riesgo es una función del nivel de activos externos netos que posea la economía y es decreciente en dicho nivel ${ }^{1}$; también depende de un componente aleatorio exógeno $\varphi$ que asume un proceso autorregresivo. Por tanto, la ecuación de la prima de riesgo es:

$$
P R_{t}=e^{-\mu\left[\left(\hat{b}_{t}-\bar{b}\right)-\varphi_{t}\right]}
$$

El incremento en los activos externos reduce la prima de riesgo, porque una mejora en la posición de la cuenta corriente reduce las necesidades de financiamiento externo. Un caso, por ejemplo, es el menor déficit fiscal, el cual eleva el ahorro interno y lleva a que sea menos necesario el ahorro externo. El otro aspecto es el shock asociado a una modificación en el apetito por el riesgo, donde un mayor apetito hará más atractiva la inversión en el país y el riesgo país disminuirá.

La prima de riesgo se incluye en la paridad descubierta de las tasas de interés, en un marco de relaciones linealizables, de la siguiente manera:

$$
\frac{\left(1+i_{t}\right)}{\left(1+i_{t}^{*}\right)}=P R_{t}\left(\frac{E_{t}\left[\epsilon_{t+1}\right]}{\epsilon_{t}}\right) .
$$

$1 P R^{\prime}(\cdot)_{t}<0$ y $P R_{t}^{\prime \prime}(\cdot)>0$. 
Linealizando la paridad de la tasa de interés, se tiene que:

$$
\hat{i}_{t}-\hat{i}_{t}^{*}=\widehat{p r}_{t}+E_{t}\left[\widehat{\varepsilon}_{t+1}\right]-\widehat{\varepsilon}_{t} .
$$

Se deduce a través de la condición $\widehat{\varepsilon}_{t}=\hat{s}_{t}-\hat{p}_{t}^{*}+\hat{p}_{d, t}$ que $\hat{i}_{t}=$ $\hat{i}_{t}^{*}+\widehat{p r}_{t}+\left(E_{t}\left[\hat{s}_{t+1}\right]-\hat{s}_{t}\right)+E_{t}\left[\pi_{d, t+1}\right]-E_{t}\left[\pi_{t+1}^{*}\right]$; es decir, la tasa de interés interna depende de la tasa de interés extranjera (libre de riesgo), la prima de riesgo y la depreciación esperada de la moneda.

Existen otras maneras análogas de modelar la prima de riesgo en la literatura de los modelos DSGE. Una de las más usuales es utilizar la prima de riesgo como respuesta del spread a cambios en la variación porcentual de la relación de endeudamiento respecto del PIB en su estado estacionario y la depreciación de la moneda. Esta definición alterna de la prima de riesgo no es muy utilizada por las calificadoras de riesgo, por lo que se modeló de la manera más tradicional y estándar dentro de la definición dada en la literatura.

\section{El banco central}

El principal instrumento del banco central es la tasa de interés de corto plazo, con la cual se afecta el comportamiento de la tasa de interés del mercado interbancario. A su vez, el tipo de interés interbancario influye en todos los tipos de interés de los créditos a corto plazo. Se supondrá que la autoridad monetaria desea estabilizar el producto alrededor de su nivel potencial, en línea con el objetivo de inflación, y por ello se especifica una regla de política monetaria tipo Taylor. Con este objetivo se propone una regla convencional de política monetaria, en la cual el banco ajusta la tasa de interés ante desviaciones de la inflación respecto de su meta, y, al mismo tiempo, responde a la desviación del producto respecto de su producto potencial (producto de estado estacionario). Además, se suaviza la tasa de intervención de acuerdo a la tasa de interés del periodo pasado. En particular, se asume la siguiente forma ecuacional:

$$
\frac{\left(1+i_{t}\right)}{\left(1+i_{t-1}\right)^{f_{i}}}=\bar{r} \pi_{t}\left(\frac{\pi_{t}}{\bar{\pi}}\right)^{f_{\pi}}\left(\frac{Y_{t}}{\bar{Y}}\right)^{f_{Y}} .
$$


Gil-León y Suárez-Cante: Implicaciones de los choques de prima de riesgo en una economía...

Su expresión linealizada es:

$$
\hat{i}_{t}=\hat{r}+\widehat{\pi}_{t}+f_{i} \hat{i}_{t-1}+f_{\pi}\left(\widehat{\pi}_{t}-\widehat{\pi}\right)+f_{Y}\left(\hat{y}_{t}-\hat{y}\right) .
$$

\section{E. Acumulación de los activos}

Los hogares domésticos y los hogares externos pueden invertir parte de sus excedentes en bonos de la economía interna, por lo que se plantea una expresión que represente el hecho de que, la acumulación o desacumulación de activos depende tanto de la producción doméstica en el periodo anterior como del consumo total, también en el periodo anterior. Entonces, de acuerdo con la expresión propuesta por Vallejos y Navia (2016) la ecuación de la dinámica de activos es:

$$
B_{t}=\left(\frac{1+i_{t-1}}{1+\pi_{t-1}}\right) B_{t-1}+P_{d, t} Y_{t-1}-P_{T, t-1} C_{T, t-1} .
$$

Los hogares acumulan activos de acuerdo al nivel de ahorro que poseen en el mismo periodo, e incrementan el nivel cuando la tasa de interés de los títulos en el periodo pasado es alta. Por tanto, la tasa de interés real incide positivamente en la acumulación de riqueza financiera.

Linealizando esta expresión por el método de Uhilg se obtiene:

$$
\hat{b}_{t}=\left(1+\hat{i}_{t-1}-\widehat{\pi}_{t-1}\right) \hat{b}_{t-1}-\left(\hat{p}_{T, t-1}-\hat{p}_{d, t}\right)+\hat{y}_{t-1}-\hat{c}_{T, t-1} .
$$

\section{F. Inversión y capital}

\section{Inversión}

Se considera que la función de inversión establece una dependencia directa de la producción $Y$ y una relación inversa con el tipo de interés $i^{2}$ Además, como el modelo desarrollado se encuentra en forma lineal, se

2 La función de inversión es una estructura de la forma $I N=f\left(\begin{array}{cc}Y & i \\ + & -\end{array}\right)$, donde el signo positivo situado debajo de $Y$ indica que un aumento de la producción provoca un incremento de la inversión, y el signo negativo que figura debajo del tipo de interés $i$ indica que una subida del tipo de interés provoca una reducción de la inversión. 
supondrá que la inversión en existencias es igual a cero, pues al momento de pasar a log-desviaciones la inversión debe fluctuar alrededor de su estado estable, por lo que las ventas y la producción siempre son iguales.

$$
I N_{t}=i_{T, t}^{-\vartheta} Y_{t-1}^{1+\vartheta}
$$

El tipo de interés en una economía abierta y dependiente del exterior en cierto grado se define como una agregación CES (Constant Elasticity of Substitution) a partir del tipo de interés doméstico y del tipo de interés externo:

$$
i_{T, t}=\left[(1-\alpha)\left(i_{t}\right)^{1-e}+\alpha\left(i_{t}^{*}\right)^{1-e}\right]^{\frac{1}{1-e}}
$$

Esta expresión linealizada por una aproximación de Taylor de orden 1 es:

$$
\widehat{i n}_{t}=-\vartheta(1-\alpha) \hat{i}_{t}-\vartheta \alpha \hat{i}_{t}^{*}+(1+\vartheta) \hat{y}_{t-1}
$$

\section{Capital}

El capital es un stock constituido por máquinas y las plantas existentes en la economía en un momento del tiempo. Suponiendo entonces que el stock de capital se mide a comienzos de cada periodo de tiempo $t$, por lo que $K_{t}$ es el stock de capital existente a comienzos del periodo $t, K_{t+1}$ es el stock de capital existente a comienzos del periodo $t+1$.

Ahora, como el capital se deprecia a una tasa $\sigma$ en cada periodo $t$, una proporción $(1-\sigma) K_{t}$ del stock de capital permanece intacta de un año a otro. Por lo tanto, la formación de capital seguirá la forma:

$$
K_{t+1}=(1-\sigma) K_{t}+I N_{t}
$$

Entonces, la forma linealizada es: $\hat{k}_{t+1}=(1-\sigma) \hat{k}_{t}+\sigma \widehat{i n}_{t}$. 
Gil-León y Suárez-Cante: Implicaciones de los choques de prima de riesgo en una economía...

\section{G. Sector externo}

El sector externo estará compuesto por las importaciones y las exportaciones; en consecuencia, para incorporar estos agentes, se asume que el consumidor demanda bienes nacionales e importados. Con la finalidad de obtener las cantidades óptimas de ambos tipos de consumo, se deduce la ecuación linealizada del consumo importado: $\widehat{i m}_{t}=-e(1-\alpha) \hat{s}_{t}+\hat{c}_{T, t}$. En el caso de las exportaciones, se considera el hecho de que depende de las cuotas de mercado junto con un componente fijo, tal como se muestra en Mickelsson (2009): $\frac{X_{t}}{Y_{t}^{*}}=\left(\alpha^{*} S_{t}^{e^{*}}\right)^{\phi}\left(\frac{X_{t-1}}{Y_{t-1}^{*}}\right)^{1-\phi}$.

La balanza comercial se define como las exportaciones menos las importaciones, $\widehat{x n}_{t}=\frac{X}{X N} \hat{x}_{t}-\frac{I M}{X N} \widehat{i m}_{t}$, y en estado estacionario se tiene que $X=I M$; además $X N=Y$, con lo cual $\frac{X}{X N}=\alpha$, entonces la ecuación final será $\widehat{x n}_{t}=\alpha\left(\hat{x}_{t}-\widehat{i m}_{t}\right)$.

\section{H. La condición de equilibrio}

La condición de equilibrio para la economía viene dada por el consumo doméstico, la inversión y la balanza comercial: $Y_{t}=C_{d, t}+I N_{t}+X N_{t}$. Esta misma ecuación en desviaciones respecto del estado estacionario es $\hat{y}_{t}=(1-\alpha) \hat{c}_{d, t}+\alpha \widehat{x n}_{t}+\tau \widehat{i n}_{t}$, donde se incluye $\tau$ como la sensibilidad de la desviación del PIB de su estado estacionario a la inversión.

\section{Estructura final del modelo}

Las condiciones de primer orden y las relaciones establecidas para el planteamiento del modelo se linealizaron. Es de aclarar que las variables deben ser trabajadas en log-desviaciones respecto del estado estacionario, y esta estructura resumida se presenta en la Tabla 2. 
Tabla 2. Sistemas de ecuaciones que componen el modelo

\begin{tabular}{|c|c|c|}
\hline \multicolumn{3}{|l|}{ Consumo } \\
\hline$\hat{c}_{T, t}=(1-\alpha) \hat{c}_{d, t}+\alpha \hat{c}_{i m, t}$ & Consumo total & E1 \\
\hline$\hat{c}_{d, t}=e \alpha \hat{s}_{t}+\hat{c}_{T, t}$ & Consumo doméstico & E2 \\
\hline \multicolumn{3}{|l|}{ Inflación y tipo de cambio real } \\
\hline$\widehat{\pi}_{t}=\widehat{\pi}_{d, t}+\alpha\left(\hat{s}_{t}-\hat{s}_{t-1}\right)$ & Inflación total & E3 \\
\hline$\hat{s}_{t}(1-\alpha)=\widehat{t c r}_{t}$ & Tasa de cambio real & E4 \\
\hline \multicolumn{3}{|l|}{ Banco central } \\
\hline$\hat{i}_{t}=r+\widehat{\pi}+f_{i} \hat{i}_{t-1}+f_{\pi}\left(E\left[\widehat{\pi}_{t+1}\right]-\widehat{\pi}\right)+f_{Y}\left(\hat{y}_{t}-\hat{y}\right)$ & Política monetaria (Regla Taylor) & E5 \\
\hline \multicolumn{3}{|l|}{ Acumulación de activos } \\
\hline$\hat{b}_{t}=\left(1+\hat{i}_{t-1}-\widehat{\pi}_{t-1}\right) \hat{b}_{t-1}-\alpha \hat{s}_{t}+\hat{y}_{t-1}-\hat{c}_{T, t-1}$ & Dinámica de inversión en bonos & E6 \\
\hline \multicolumn{3}{|l|}{ Paridad de tasas de interés y prima de riesgo } \\
\hline$\widehat{p r}_{t}=-\mu\left[\left(\hat{b}_{t}-\bar{b}\right)-\varphi_{t}\right]$ & Prima de riesgo & E7 \\
\hline$\hat{i}_{t}-\hat{i}_{t}^{*}=\widehat{p r}_{t}+\left(E_{t}\left[\hat{s}_{t+1}\right]-\hat{s}_{t}\right)+E_{t}\left[\pi_{d, t+1}\right]-E_{t}\left[\pi_{t+1}^{*}\right]$ & Paridad de tasas de interés & E8 \\
\hline \multicolumn{3}{|l|}{$\begin{array}{ll}\text { Hogares } \\
\end{array}$} \\
\hline$\hat{w}_{t}=\gamma \hat{l}_{t}+\frac{\ni}{1-\eta}\left(\hat{c}_{T, t}-\eta \hat{c}_{T, t-1}\right)$ & Función de oferta laboral & E9 \\
\hline$\frac{-\ni}{1-\eta}\left(\hat{c}_{T, t}-\eta \hat{c}_{T, t-1}\right)=\frac{-\ni}{1-\eta} E_{t}\left[\left(\hat{c}_{T, t+1}-\eta \hat{c}_{T, t}\right)\right]+\hat{i}_{t}-E_{t}\left[\widehat{\pi}_{t+1}\right]$ & Curva IS dinámica & E10 \\
\hline \multicolumn{3}{|l|}{ Inversión y capital } \\
\hline$\hat{i n}_{t}=-\vartheta(1-\alpha) \hat{i}_{t}-\vartheta \alpha \hat{i}_{t}^{*}+(1+\vartheta) \hat{y}_{t-1}$ & Función de inversión & E11 \\
\hline$\hat{k}_{t+1}=(1-\sigma) \hat{k}_{t}+\sigma \widehat{i n}_{t}$ & Función de capital & E12 \\
\hline \multicolumn{3}{|l|}{ Empresas } \\
\hline$\hat{y}_{t}=\hat{a}_{t}+\delta \hat{k}_{t}+(1-\delta) \hat{l}_{t}$ & Función de producción & E13 \\
\hline $\mathrm{e} \widehat{\pi}_{d, t}=\beta E_{t}\left[\widehat{\pi}_{d, t+1}\right]$ & Curva de Phillips Neokeynesiana & E14 \\
\hline$+\rho\left[\frac{(\gamma+\delta)}{(1-\delta)} \hat{y}_{t}-\frac{(\gamma+1)}{(1-\delta)} \hat{a}_{t}-\frac{(\gamma+1) \delta}{(1-\delta)} \hat{k}_{t}+\frac{\ni}{1-\eta}\left(\hat{c}_{T, t}-\eta \hat{c}_{T, t-1}\right)+\alpha \hat{s}_{t}\right]$ & 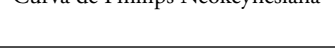 & 517 \\
\hline \multicolumn{3}{|l|}{ Sector externo } \\
\hline$\hat{x}_{t}=\hat{y}_{t}^{*}+\phi e^{*} \hat{s}_{t}+(1-\phi)\left(\hat{x}_{t-1}-\hat{y}_{t-1}^{*}\right)$ & Función de exportaciones & E15 \\
\hline$\widehat{x m}_{t}=\alpha\left(\hat{x}_{t}-\widehat{i m}_{t}\right)$ & Función de balanza comercial & E16 \\
\hline \multicolumn{3}{|l|}{ Equilibrio } \\
\hline$\hat{y}_{t}=(1-\alpha) \hat{c}_{d, t}+\alpha \widehat{x n}_{t}+\tau \widehat{i n}_{t}$ & PIB & E17 \\
\hline \multicolumn{3}{|l|}{ Choque autorregresivo } \\
\hline$\varphi_{t}=\chi_{r} \varphi_{t-1}+e_{r}$ & Shock de prima de riesgo & E18 \\
\hline$a_{t}=\chi_{a} a_{t-1}+e_{a}$ & Shock tecnológico & E19 \\
\hline
\end{tabular}

Fuente: elaboración propia.

Lecturas de Economía -Lect. Econ. - No. 92. Medellín, enero-junio 2020 
Gil-León y Suárez-Cante: Implicaciones de los choques de prima de riesgo en una economía...

\section{Calibración del modelo}

La calibración del modelo incorpora información de la economía colombiana entre el primer trimestre de 2005 y cuarto trimestre de 2017. Se establece el estado estacionario en el cual se elimina el parámetro temporal de las variables del modelo, y de esta forma es posible despejar de las ecuaciones resultantes los parámetros fundamentales y encontrar su valor a partir de la evidencia empírica de las series temporales observadas. Algunos parámetros son establecidos a partir de una colección de artículos similares, donde se exponen condiciones económicas y modelos DSGE para Colombia que hacen referencia a aspectos semejantes a los de este trabajo. La tabla 3 muestra el resumen de los parámetros calibrados.

Los parámetros involucrados para el consumo son: $\alpha$ es el grado de apertura de la economía nacional con el resto del mundo. Este indicador se define como el porcentaje que supone los flujos del comercio exterior (exportaciones e importaciones en el total del PIB); es decir, $\alpha=\frac{\bar{X}+\overline{I M}}{\bar{Y}}$, el cual es 0,4 tomando datos del Banco de la República de Colombia correspondientes al año 2017. Además, $e$ es la elasticidad de sustitución entre bienes de consumo nacionales e importados (valor tomado de Suescún y Steiner, 2017).

Los parámetros involucrados para el banco central son tomados del Banco de la República, donde $\bar{r}$ es el logaritmo de la tasa de interés real esperada y, según el Banco de la República, para Colombia es $2 \%$. Según Gil (2015), el peso de la brecha de la inflación en la reacción de la tasa de política es 1,5 y el peso de la brecha PIB a la reacción de política es 0,5 . Finalmente, de acuerdo al comunicado de 2017 del Banco de la República, el PIB potencial para Colombia crece 3,5\%.

Por el lado de los parámetros de la paridad de tasas de interés y prima de riesgo, la elasticidad de la prima de riesgo con respecto al endeudamiento externo $\mu$ es tomado de Suescún y Steiner (2017). El indicador más utilizado para medir la prima de riesgo en mercados emergentes es el EMBIG (Emerging Markets Bonds Index Global), medido por el banco JP Morgan Chase. En la figura 1 se observa el comportamiento del indicador de prima de riesgo, el cual 
se eleva en momentos de incertidumbre cuando el apetito por el riesgo de los inversiones se reduce (posterior a las crisis de 2018 se eleva) o cuando existe una mayor presión desde la cuenta corriente (el deterioro de los términos de intercambio eleva la prima de riesgo).

Tabla 3. Valores de los parámetros del modelo

\begin{tabular}{|c|c|c|}
\hline Origen & Parámetro & Valor \\
\hline \multirow{2}{*}{ Consumo } & $\alpha$ & 0,4000 \\
\hline & $e$ & 1,5000 \\
\hline \multirow{6}{*}{ Banco central } & $\hat{r}$ & 0,0198 \\
\hline & $f_{i}$ & 1,0070 \\
\hline & $f_{\pi}$ & 1,5000 \\
\hline & $f_{Y}$ & 0,5000 \\
\hline & $\widehat{\pi}$ & 0,0295 \\
\hline & $\hat{y}$ & 0,0344 \\
\hline \multirow{2}{*}{ Paridad de tasas de interés y prima de riesgo } & $\mu$ & 0,4510 \\
\hline & $\hat{b}$ & 0,0342 \\
\hline \multirow{4}{*}{ Hogares } & $\gamma$ & 1,6000 \\
\hline & $\ni$ & 2,0000 \\
\hline & $\eta$ & 0,2572 \\
\hline & $\beta$ & 0,9456 \\
\hline \multirow{2}{*}{ Inversión y capital } & $\sigma$ & 0,0287 \\
\hline & $\vartheta$ & 0,2965 \\
\hline \multirow{2}{*}{ Empresas } & $\delta$ & 0,5963 \\
\hline & $\rho$ & 0,7500 \\
\hline \multirow{2}{*}{ Sector externo } & $\phi$ & 0,1000 \\
\hline & $e^{*}$ & 2,1400 \\
\hline Equilibrio & $\tau$ & 0,0074 \\
\hline \multirow{4}{*}{ Choque autorregresivo } & $\chi_{a}$ & 0,8600 \\
\hline & $\sigma_{a}^{2}$ & 0,1000 \\
\hline & $\chi_{r}$ & 0,7610 \\
\hline & $\sigma_{r}^{2}$ & 0,1000 \\
\hline
\end{tabular}

Fuente: elaboración propia. 
Gil-León y Suárez-Cante: Implicaciones de los choques de prima de riesgo en una economía...

Figura 1. Comportamiento del EMBIG

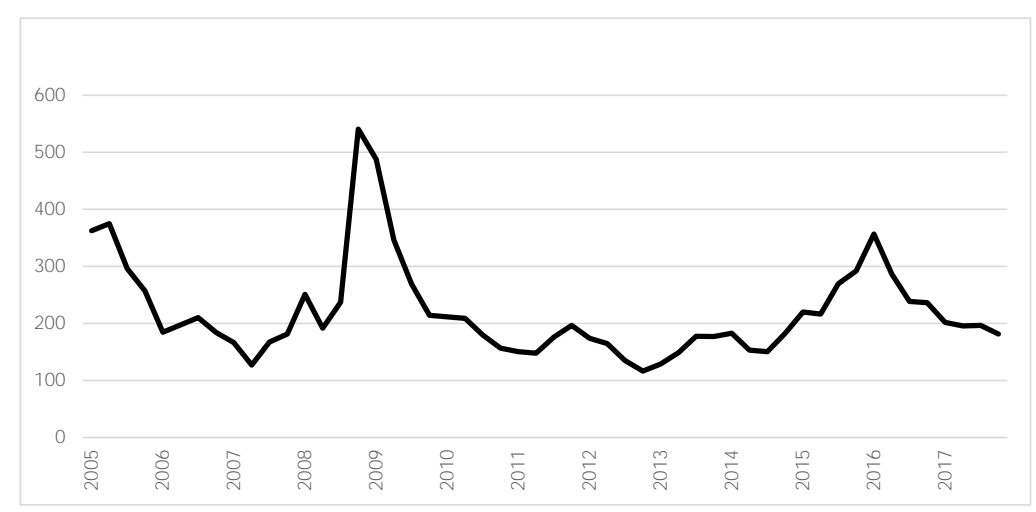

Fuente: datos del JP Morgan Chase (2018).

Para la calibración se considera el promedio del EMBIG, que es equivalente a 0,02204 (220 puntos básicos), por lo que se reescribe en estado estacionario como $\widehat{p r}=\ln (1+E m b i g)$. Ahora, en la ecuación (8) se considera $\hat{b}_{t}$ como la tasa promedio de los títulos de deuda pública interna (TES) a 10 años $(\hat{b}=\ln (1+T e s))$.

Los parámetros de las ecuaciones de los hogares se toman de diferentes estudios, $\gamma$ y $\ni$ son calculados por Hernández (2013), $\eta$ determinado por Bonaldi, González y Rodríguez (2010), y $\beta$ se deduce del estado estacionario $\beta=\frac{1}{1+i}$, donde $i=0,0575$ es el promedio del tipo de interés fijado por el Banco de la República.

De otro lado, la tasa de depreciación física del capital $\sigma$ es tomada de Hernández (2013), donde $\vartheta=\frac{\ln (Y)-\ln (\sigma K)}{\ln \left(i_{T}\right)-\ln (Y)}$. Para poder realizar el cálculo se requiere la ecuación $i_{T}=\left[(1-\alpha)(i)^{1-e}+\alpha\left(i^{*}\right)^{1-e}\right]^{\frac{1}{1-e}}$ a partir de $i=0,0575$ y $i^{*}=0,015$, que se toma de la Reserva Federal de Estados Unidos. Además, el parámetro $\delta$ es determinado por Bonaldi et al. (2010), y $\rho$ por Hernández (2013).

Los parámetros del sector externo son: $\phi$ es la elasticidad de las exportaciones frente a la economía externa, y será de 0,1 y $e^{*}$ es la elasticidad 
de la sustitución de bienes del extranjero, datos tomados de Santana (2016). Finalmente, $\tau=\frac{\sigma K}{Y}$, donde $\sigma=0,0287, K=117.276$ y $Y=452.592$.

Por último, los parámetros de persistencia de los choques siguen un proceso autoregresivo de orden uno para la prima de riesgo y uno de orden dos para la tecnología. Se toma de Hernández (2013) la persistencia de ajuste al shock tecnológico, que es 0,86 y su desviación estándar, que es 0,1 . La persistencia del shock de prima de riesgo se estima mediante un modelo autorregresivo integrado de promedio móvil con datos trimestrales del EMBI; de lo cual se obtuvo que $\chi_{r}=0,761$ y una desviación estándar de 0,1 . Esto se hace sobre la especificación econométrica de cada una de las variables, que en este modelo corresponde a las versiones log-lineales.

\section{Análisis de resultados}

El modelo se resolvió de manera lineal en Dynare. ${ }^{3}$ Allí todas las variables son medidas como desviaciones de su tendencia, la cual se obtuvo mediante el filtro de Hodrick-Prescott con un parámetro de suavización de 1.600, indicando con esto que los periodos de análisis en los resultados y funciones de impulso-respuesta están dados en trimestres. Las gráficas de impulsorespuesta trazan la réplica de cada variable al choque en prima de riesgo y de las implicaciones de los choques tecnológicos (incluidos en los anexos). Las Figuras 2 y 3 muestran los resultados en términos de desviaciones del estado estable para cada una de las variables (en puntos porcentuales trimestrales).

Para este artículo se planteó un shock positivo del $10 \%$ sobre la prima de riesgo (Figura 2). Como dicha prima es un componente de la variedad de las tasas de interés, entonces este choque impacta inicialmente la tasa de interés interna, lo que a su vez hace que esta varíe, inicialmente, en una proporción del $3,3 \%$ respecto a su estado estacionario (Figura 3). Este impacto es menor al choque inicial en la prima de riesgo, debido a las características de la economía

3 Dynare es una plataforma de software con un conjunto de herramientas diseñadas para manejar una amplia clase de modelos económicos, en particular modelos DSGE y generaciones. Los modelos resueltos por Dynare incluyen la hipótesis de expectativas racionales para los agentes económicos, en donde los agentes forman sus expectativas sobre el futuro de una manera consistente con el modelo. 
Gil-León y Suárez-Cante: Implicaciones de los choques de prima de riesgo en una economía...

colombiana, ya que esta presenta un grado de elasticidad en prima de riesgo relativamente bajo — menos de 0,5 - respecto al exterior. Luego la tasa de interés presenta una disminución gradual, hasta que en el cuarto y quinto trimestre de esta disminución llega a ser máxima y alcanza una variación de alrededor del $-0,4 \%$. El resultado se debe a que los efectos del shock sobre la prima de riesgo se tornan negativos después del cuarto trimestre, hasta volver al estado estacionario; a partir del quinto trimestre el tipo de interés comienza una recuperación gradual, por lo que alcanza su estado de equilibrio alrededor del trimestre doce, esto en virtud del hecho de que el riesgo ahora es menor y se estabiliza alrededor del mismo trimestre.

Figura 2. Proceso de ajuste de un shock en prima de riesgo
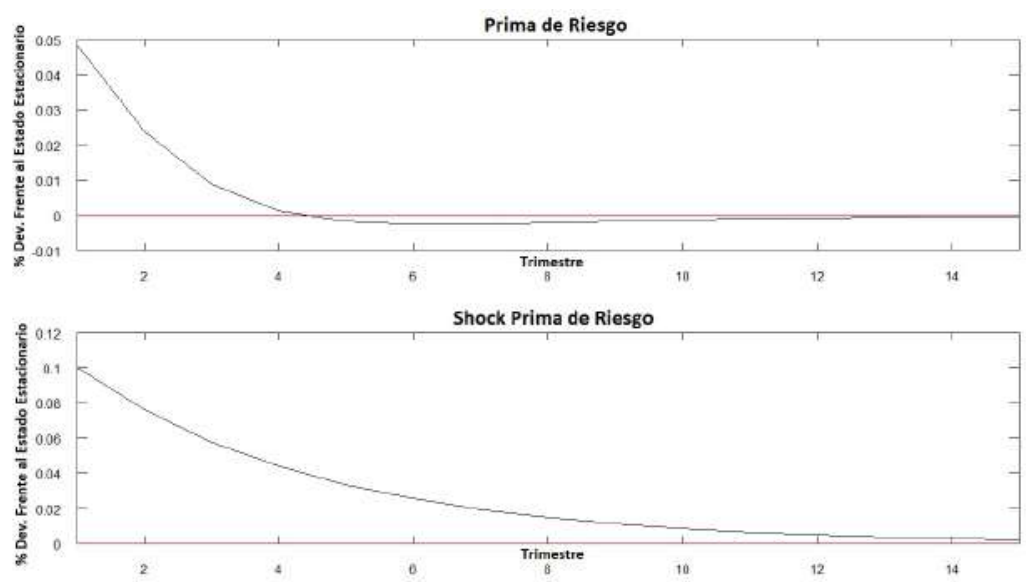

Fuente: elaboración propia.

Ahora, como las tasas de interés nominales en el país y en el exterior están determinadas por las respectivas reglas de política monetaria, la prima de riesgo tiene un efecto principal en la tasa de cambio y en los términos de intercambio, debido principalmente a la existencia de un escenario de tipos de cambio flexible y movilidad de capitales dentro de la economía colombiana; por lo tanto, el shock positivo en prima de riesgo genera que, inicialmente, los términos de intercambio se eleven positivamente alrededor del 1,75\% (Figura 2), y que luego desciendan rápidamente hasta el quinto trimestre, 
punto de inflexión donde se presenta una recuperación hasta alcanzar su estado de equilibrio en el trimestre doce. El trasfondo económico detrás de este hecho es que el aumento en la prima de riesgo país produce una depreciación de la moneda nacional, como se observa, y dicha depreciación actúa como un amortiguador (López et al., 2008). La situación mencionada también desfavorece a las importaciones, ya que los términos de intercambio aumentan. Lo anterior implica directamente que los precios de importación aumenten, y esto se refleja en una disminución alrededor del 3,75\% en las importaciones. Es de resaltar que el consumo importado presenta una recuperación muy rápida, tal como se evidencia en la pendiente de la gráfica de impulso respuesta; tal es así que a partir del tercer y cuarto trimestre alcanza su estado estacionario y se genera un aumento máximo en las importaciones del $1 \%$ alrededor del quinto trimestre, tendiendo nuevamente a estabilizarse alrededor del doceavo trimestre, como es tendencia en el modelo.

Figura 3. Transmisión del shock en prima de riesgo sobre las variables
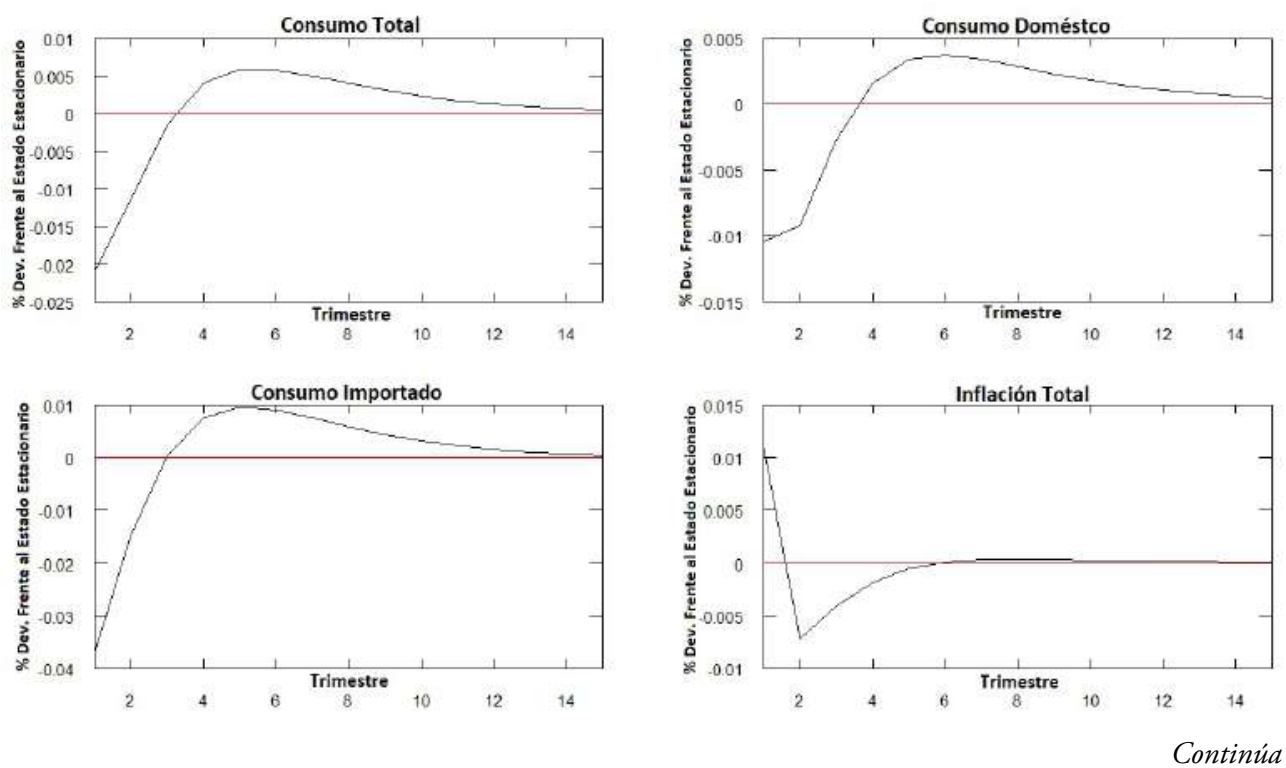
Gil-León y Suárez-Cante: Implicaciones de los choques de prima de riesgo en una economía...

Figura 3. Continuación
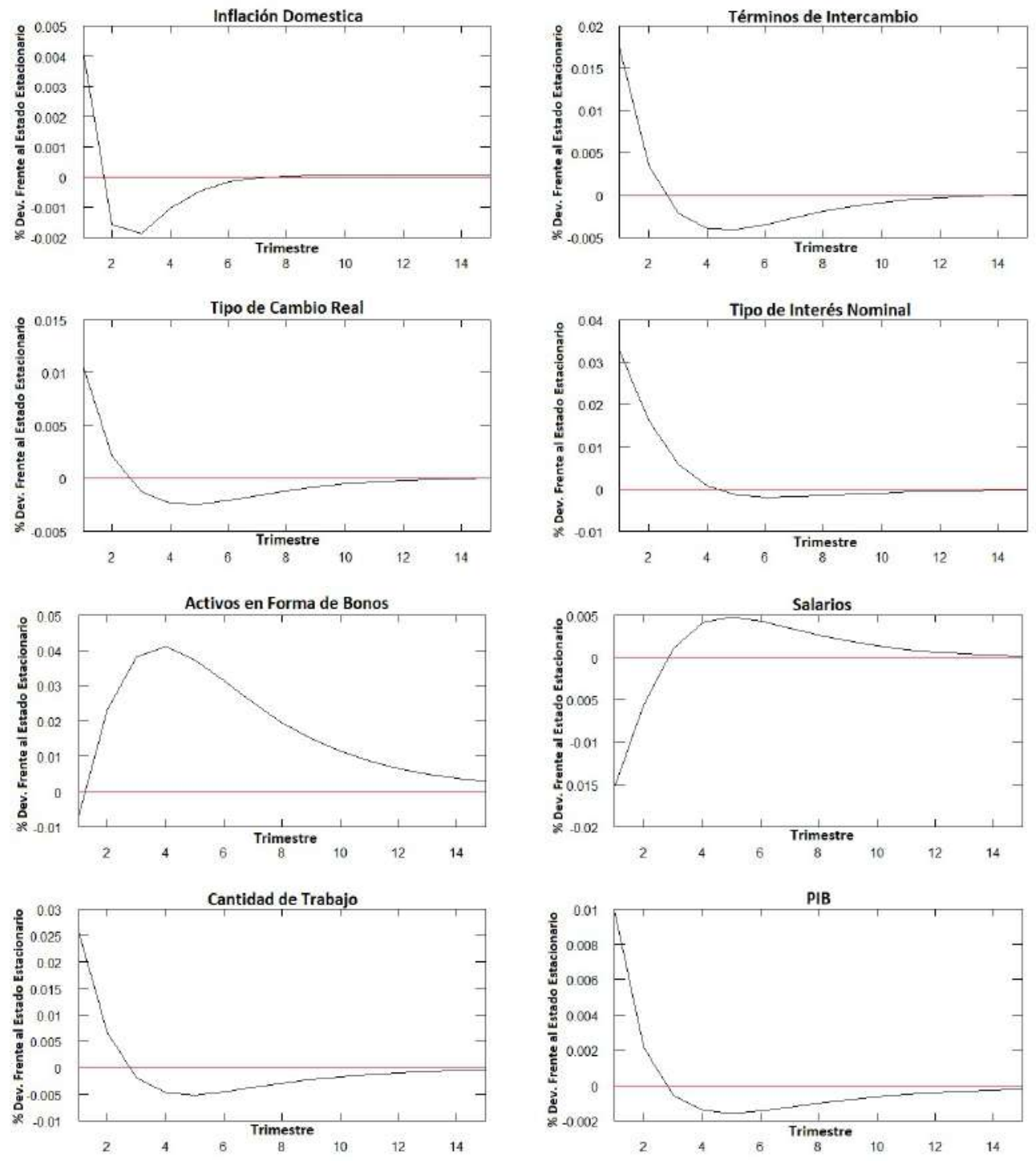

Continúa 
Figura 3. Continuación
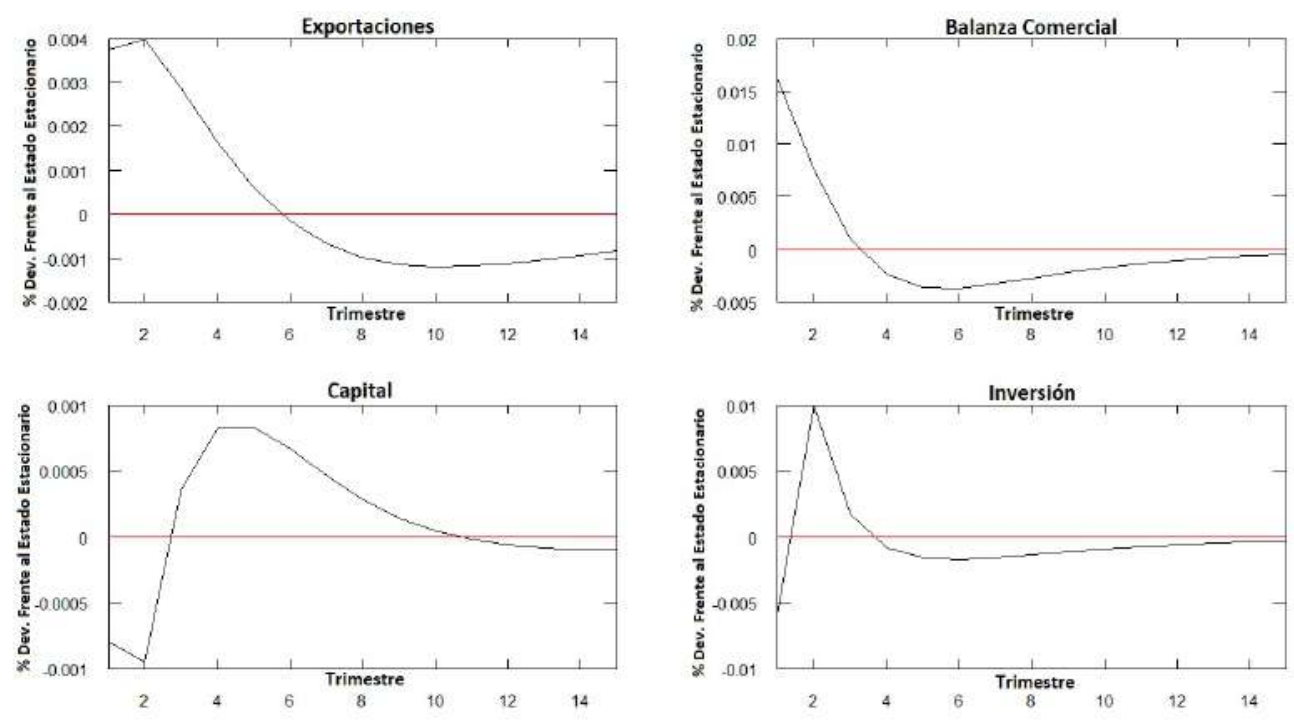

Fuente: elaboración propia.

Por otro lado, la depreciación de la moneda, reflejada en el aumento de los términos de intercambio, genera un favorecimiento en las exportaciones, que en este caso particular es, inicialmente, de alrededor del 0,38\%, posteriormente alcanza su máximo de $0,4 \%$ en el segundo trimestre, donde se está presentando una tendencia decreciente y se mantiene siempre inferior a su estado de equilibrio. Esto, en conjunto con las importaciones, primero genera un aumento del 1,7 \% en la balanza comercial (Figura 3), y luego genera una reducción gradual en esta hasta el quinto trimestre del $-0,4 \%$, para que después tienda hacia su estado de equilibrio alrededor del trimestre catorce.

Como ya se mencionó, los shocks en prima de riesgo tienen un papel importante en el comportamiento de la tasa de interés, y estas variaciones se han relacionado con movimientos directos que se reflejan en las tasas de inflación (Rudolf \& Zurlinden, 2014). En este modelo se evidencia que la inflación total presenta una variación de alrededor 1,2\%, y la inflación doméstica una variación del 0,4\%; esto muestra una disminución gradual de dichas tasas hasta el segundo y tercer trimestre luego de los cuales entran 
Gil-León y Suárez-Cante: Implicaciones de los choques de prima de riesgo en una economía...

en un periodo de recuperación y tienden a su estado estacionario a partir del sexto trimestre. Esto es debido a que inicialmente el shock genera movimientos por encima de la media (Soares, Portugal \& da Silva Bejarano, 2016), lo que indica que la política monetaria responde elevando las tasas de interés, pero con el deterioro gradual del shock a partir del primer trimestre se genera una recuperación acelerada de dichas tasas como respuesta a la política acelerada de meta de inflación que se tiene para Colombia.

En lo referente al consumo total y al consumo doméstico se presenta una disminución en su variación porcentual alrededor del -2,1\% y del -1,2\%, respectivamente (mucho menor que la variación del consumo importado); luego, presenta una recuperación gradual hacia el quinto trimestre del 0,5\% y del $0,4 \%$. A partir de este punto crítico las curvas presentan una tendencia decreciente a sus estados estacionarios respectivos alrededor del trimestre catorce para ambos casos. Este comportamiento es muy usual, y se debe, en primer lugar, a que los modelos con presencia de rigideces de precios típicamente conducen a un menor pass-through en el consumo doméstico (Bergholt, 2015), y, en segundo lugar, al ajuste en la tasa de interés, debido a que el shock en prima de riesgo genera una forma de campana asimétrica positiva (Figura 3). Este perfil de consumo es dado, principalmente, por un comportamiento inercial generado por la rigidez de precios y salarios (tal como se presentan en la economía colombiana), lo que refleja la incapacidad de pasar rápidamente del consumo al ahorro en respuesta a la variación repentina en el tipo de interés. Como consecuencia, la demanda de todo tipo de bienes en la canasta de consumo disminuye, particularmente la de productos extranjeros.

El shock señalado induce también a un aumento en la producción, lo que genera un aumento en la cantidad de trabajo requerido en la economía; a su vez el shock en prima de riesgo genera una percepción de deterioro de la economía doméstica y conduce a una salida de capital financiero. Lo anterior se refleja en la depreciación de la moneda y en el aumento de la tasa de interés a largo plazo, lo que dificulta el consumo; sin embargo, la depreciación mejora las exportaciones netas, y esto resulta en un crecimiento del PIB y, como consecuencia, en un aumento de la inflación. En ese sentido, la desviación de la inflación de su meta ocasiona que el banco central eleve las tasas de 
interés a corto plazo para limitar así el crecimiento del PIB y presionar al descenso de la inflación. Lo anterior ocasiona que la producción y la cantidad de trabajo disminuyan rápidamente hasta el quinto semestre, y a partir de este punto comienza una mejora que les permite a las variables tender hacia su estado estacionario. Similar a los resultados de Bergholt (2015), a partir del trimestre catorce existe esta misma tendencia para la mayoría de las variables, debido a que la menor demanda de consumo incide en la inflación total. Esto es particularmente relevante para las empresas, las cuales suministran la mayoría de los bienes y servicios de consumo nacional, y por esto afectan el PIB. De hecho, la disminución agregada en la absorción doméstica es lo suficientemente grande como para contraer el PIB y, por ende, la demanda de trabajo. Sin embargo, con la simple observación del proceso optimizador las empresas ajustan los precios, y con esto pueden responder de manera adecuada a la depreciación que se genera inicialmente (Amano \& Shukayev, 2012). Esto significa que la dinámica de pass-through es incompleta en las empresas.

De igual manera, un aumento en la prima de riesgo, causado por el shock positivo, provoca una depreciación de la moneda (aumento en la tasa de cambio) y, como ya se dijo, esto conduce a un aumento en la relación de intercambio. A su vez, dicho aumento produce una expansión en los ingresos externos por exportaciones y una disminución en la inversión (Posch, 2009). En ese sentido, se evidencia que, debido al shock, el riesgo aumenta alrededor de 5 puntos porcentuales, lo que origina una mayor probabilidad de default, con lo cual la prima de riesgo se reduce. Sin embargo, esta se mantiene por encima del estado estacionario en los primeros cuatro trimestres, y desciende muy poco hasta recuperase y situarse en su estado estacionario anterior. Esto conduce una dinámica en la cual se disminuye el financiamiento externo, debido también, en cierto grado, a las rigideces en los precios de la economía. Pero no es rentable para los inversionistas otorgar recursos, al reducir los ingresos aproximadamente en los primeros trimestres, presionando también la caída en la formación de capital. Por lo tanto, la dinámica después del impacto inicial es así: el crecimiento del PIB es menor y converge al estado estacionario anterior al shock positivo sobre la prima de riesgo, debido a una 
Gil-León y Suárez-Cante: Implicaciones de los choques de prima de riesgo en una economía...

tasa de interés interna más alta generada por el choque, lo que reduce el consumo interno, la demanda de inversión y con esto la formación de capital.

Figura 4. Resumen del shock en prima de riesgo

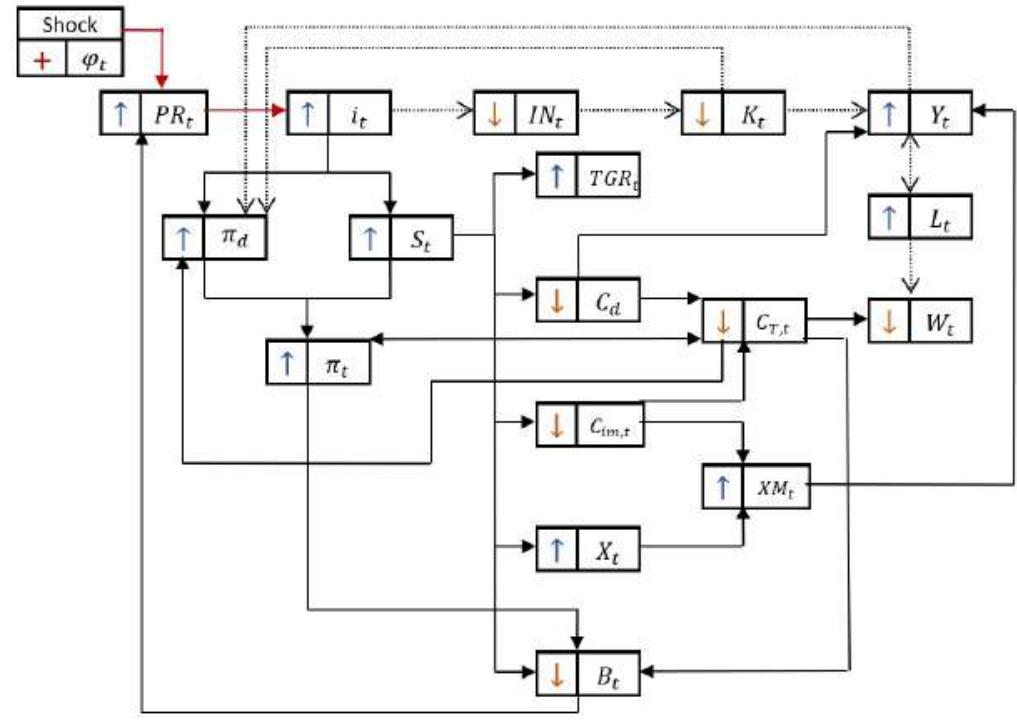

Fuente: elaboración propia.

De otra parte, el shock auxiliar tecnológico del 10\% (ver anexo 2) genera una variabilidad inicialmente positiva alrededor del 1,7\% sobre la prima de riesgo y una reducción gradual cercana a $0,55 \%$; a partir de ahí, se presenta una recuperación en la prima de riesgo con tendencia hacia su estado estacionario, el cual se alcanza después de catorce trimestres. Al mismo tiempo, se observa una disminución en la variación de la tasa de interés, que es el principal canal de transmisión de la prima de riesgo, donde dicha variación es de 3,2\%, y una posterior recuperación gradual. La explicación se justifica en lo que señalan Vallejos y Navia (2016), quienes aseguran que los shocks tecnológicos impactan al mismo tiempo la productividad, por lo que se reducen los costos marginales de estos bienes y se afecta negativamente la inflación de bienes domésticos. Por otro lado, la reducción de la inflación doméstica reduce la inflación total, lo que motiva una reducción de la tasa de interés nominal por parte del banco central. En consecuencia, cuando el 
banco central reduce la tasa de interés real, se genera una depreciación real vía la paridad de tasas de interés, y como la prima de riesgo es parte fundamental de esta, expande su variación ante una respuesta a la reducción del tipo de cambio y a la situación económica después del shock tecnológico. Por lo tanto, a partir de este hecho se eleva el valor de las exportaciones, mientras que los consumidores aumentan el consumo total.

\section{Conclusiones}

En esta investigación se formuló un modelo de equilibrio general dinámico y estocástico para la economía colombiana. El modelo posibilitó un acercamiento al impacto de un choque en prima de riesgo sobre los principales agregados macroeconómicos. La principal conclusión respecto de los resultados de este trabajo sugiere que es necesario considerar cuidadosamente los shocks de prima de riesgo, puesto que un shock se refleja en una mayor depreciación nominal y real, como también en un aumento de la tasa de política monetaria. En otras palabras, la evidencia permite concluir que para Colombia los shocks en prima de riesgo explican una parte importante de la variabilidad en la tasa de cambio. Adicionalmente, estos cambios provocan en el corto plazo una reasignación importante de recursos en todos los sectores.

La dinámica se debe a que el ajuste del tipo de cambio no absorbe todo el diferencial de tasas internas y externas creadas por el aumento de la prima de riesgo. Entonces, el aumento del tipo de interés, combinado con una depreciación nominal y real, crea unas condiciones favorables para el sector exportador. $\mathrm{Al}$ aumentar las exportaciones y disminuir las importaciones disminuye el consumo total, y como dicha disminución es menor a la de la balanza comercial se genera una brecha positiva de la producción. Por otro lado, la depreciación de la moneda impacta en los costos marginales de las empresas, lo cual disminuye el nivel de salarios. Lo anterior genera que se acumulen activos externos netos que presionan la desaparición del shock (mejora en la cuenta corriente).

Con respecto a la tendencia de las variables a su estado estacionario, se concluye que el impacto real del shock en prima de riesgo sobre la economía 
Gil-León y Suárez-Cante: Implicaciones de los choques de prima de riesgo en una economía...

es en cierto grado absorbido por todos los sectores en los primeros cuatro trimestres. A partir del cuarto trimestre se tiene un punto de inflexión donde la economía comienza a recuperarse, y alrededor de catorce trimestres después se llega al estado de equilibrio. Esto se debe al grado de dependencia de la economía colombiana a la calificación de la prima de riesgo, y, por esta razón, se debe considerar como un elemento importante la elasticidad de prima de riesgo. La reducción de la elasticidad de prima de riesgo se puede lograr mediante dos caminos principales: primero, la mayor estabilidad de las variables macroeconómicas y, segundo, mediante la mayor proporción de inversión extranjera en el país, con el fin de ampliar la liquidez de moneda extranjera.

A pesar de la amplitud en los análisis, los resultados obtenidos son consistentes con los obtenidos por autores como Posch (2009), Bergholt (2015), Ramírez y Torres (2013) y Pienika (2014) a nivel mundial, puesto que en Colombia no se ha abordado directamente los efectos de la prima de riesgo sobre los agregados macroeconómicos con modelos DSGE. Sin embargo, el modelo puede ser extendido y utilizado para analizar el comportamiento de los sectores económicos y la dinámica explícita del gobierno.

\section{Anexos}

\section{Anexo 1. Dinámica de los precios en el modelo}

\section{A. Precios de bienes importados}

Se supone que la ley de precio único es válida para bienes individuales en todo momento (tanto para los precios de importación como los de exportación). Esto implica que una familia de precios se puede transar mediante un tipo de cambio nominal bilateral (el precio de la moneda del país $r$ en términos de la moneda nacional), donde el precio del país $r$ es expresado en términos de su propia moneda $k$.

$$
P_{t}(r, k)=\epsilon_{t} P_{r, t}(r, k) \forall r, k \quad \text { con } \quad 0 \leq r, k \leq 1 .
$$

Entonces, se define al índice de precios internos del país $r$, así: 


$$
P_{r, t}(r)=\left[\int_{0}^{1} P_{r, t}(r, k)^{1-e_{d}} d r\right]^{\frac{1}{1-e_{d}}} .
$$

Expresando este índice de precios internos para el país $r$ (expresado en términos de su propia moneda) como log-desviaciones a partir de su estado estacionario, tenemos:

$$
\hat{p}_{r, t}(r)=\int_{0}^{1} \hat{p}_{r, t}(r, k) d r
$$

con lo cual es posible definir el índice de precios mundiales en log desviaciones, como:

$$
\hat{p}_{t}^{*}=\int_{0}^{1} \hat{p}_{r, t}(r) d r,
$$

y el tipo de cambio nominal bilateral del país $r$ se define así:

$$
\epsilon_{t}=\left[\int_{0}^{1} \epsilon_{t}(r)^{1-e_{i m}} d r\right]^{\frac{1}{1-e_{i m}}}
$$

Por lo tanto, se puede expresar el nivel de precios importados como:

$$
\hat{p}_{i m, t}=\int_{0}^{1}\left[\widehat{\varepsilon}_{t}(r)+\hat{p}_{r, t}(r)\right] d r
$$

Entonces se concluye que $P_{i m, t}=\epsilon_{t} P_{t}^{*}$ o, lo que es lo mismo, en su versión log-linealizada:

$$
\hat{p}_{i m, t}=\widehat{\varepsilon}_{t}+\hat{p}_{t}^{*}
$$

Remplazando esta identidad en la ecuación (3), se tiene que:

$$
\hat{s}_{t}=\widehat{\varepsilon}_{t}+\hat{p}_{t}^{*}-\hat{p}_{d, t}
$$

\section{B. Canasta de consumo}

La economía doméstica tiene dos tipos de empresas: productores nacionales e importadores y con estructura continua de cada tipo $r$. Los 
Gil-León y Suárez-Cante: Implicaciones de los choques de prima de riesgo en una economía...

productores nacionales venden sus productos a los consumidores nacionales y extranjeros, mientras que los importadores solo venden sus productos en el mercado nacional. En este sentido, el índice de consumo total utilizado en este modelo está compuesto por una canasta de consumo de bienes y servicios producidos domésticamente, como también de bienes y servicios importados, lo cual se representa mediante una función de elasticidad de sustitución constante (CES, por sus siglas en inglés). Por lo tanto, se tendrá:

\section{- Consumo doméstico}

La canasta de consumo del bien doméstico es una agregación CES de $r$ índices compuestos de diferentes variedades de bienes domésticos, dada por la siguiente ecuación:

$$
C_{d, t}=\left[\int_{0}^{1} C_{d, t}(r)^{\frac{e_{d}-1}{e_{d}}} d r\right]^{\frac{e_{d}}{e_{d}-1}}, e_{d}>1 \quad \text { y } \quad \forall r .
$$

\section{- Consumo importado}

La canasta de consumo del bien importado es una agregación CES de $r$ variedades de estos, dada por la siguiente ecuación:

$$
C_{i m, t}=\left[\int_{0}^{1} C_{i m, t}(r)^{\frac{e_{i m}-1}{e_{i m}}} d r\right]^{\frac{e_{i m}}{e_{i m}-1}}, e_{i m}>1 \quad \text { y } \quad \forall r .
$$

\section{- Consumo total}

La canasta de consumo total de la economía es nuevamente una agregación CES, tanto del bien de consumo producido domésticamente como del bien de consumo importado, ya que esta función es la mejor manera de representar una agregación indexada:

$$
C_{T, t}=\left[(1-\alpha)^{\frac{1}{e}}\left(C_{d, t}\right)^{\frac{e-1}{e}}+\alpha^{\frac{1}{e}}\left(C_{i m, t}\right)^{\frac{e-1}{e}}\right]^{\frac{e}{e-1}}, e>0 .
$$

El consumo total es linealizado a través de una expansión de Taylor de logaritmo natural alrededor de $e=0$ y de orden 1 , pues este resultado es útil en el desarrollo del modelo: 


$$
\hat{c}_{T, t}=(1-\alpha) \hat{c}_{d, t}+\alpha \hat{c}_{i m, t}
$$

Por otro lado, se tendrá que el gasto total de los hogares es:

$$
\begin{aligned}
G H_{t} & =P_{T, t} C_{T, t} \\
& =P_{d, t} C_{d, t}+\epsilon_{t} P_{t}^{*} C_{i m, t}
\end{aligned}
$$

Es así que se formula un problema de minimización del gasto del hogar:

$$
\min _{C_{T, t}} G H_{t}
$$

Sujeto a:

$$
C_{T, t}=\left[(1-\alpha)^{\frac{1}{e}}\left(C_{d, t}\right)^{\frac{e-1}{e}}+\alpha^{\frac{1}{e}}\left(C_{i m, t}\right)^{\frac{e-1}{e}}\right]^{\frac{e}{e-1}},
$$

de donde se deducen las condiciones de primer orden y se obtiene:

$$
\begin{gathered}
C_{d, t}=\frac{\lambda_{t}^{e}(1-\alpha) C_{T, t}}{\left(P_{d, t}\right)^{e}} \\
C_{i m, t}=\frac{\lambda_{t}^{e} \alpha C_{T, t}}{\left(\epsilon_{t} P_{t}^{*}\right)^{e}}
\end{gathered}
$$

Remplazando (A12) y (A13) en (A9) se tendrá en forma resumida:

$$
C_{T, t}=\lambda_{t}^{e} C_{T, t}\left[(1-\alpha)\left(P_{d, t}\right)^{1-e}+\alpha\left(\epsilon_{t} P_{t}^{*}\right)^{1-e}\right]^{\frac{e}{e-1}} .
$$

Por tanto, si se define de manera similar a (A9) los precios totales como una agregación CES se tendrá:

$$
P_{T, t}=\left[(1-\alpha)\left(P_{d, t}\right)^{1-e}+\alpha\left(\epsilon_{t} P_{t}^{*}\right)^{1-e}\right]^{\frac{1}{1-e}} .
$$


Gil-León y Suárez-Cante: Implicaciones de los choques de prima de riesgo en una economía...

\section{C. Nivel de precios para las canastas}

El índice de precios total utilizado en este modelo está compuesto por un índice de precios doméstico, como también de un índice de precios importados, lo cual se representa mediante una función de elasticidad de sustitución constante (CES).

\section{- Precios domésticos}

El nivel de precios del bien doméstico es una agregación CES de $r$ variedades de estos, dada por la siguiente ecuación:

$$
P_{d, t}=\left[\int_{0}^{1} P_{d, t}(r)^{1-e_{d}} d r\right]^{\frac{1}{1-e_{d}}}, e_{d}>1 \quad \text { y } \quad \forall r .
$$

\section{- Precios importados}

El nivel de precios del bien importado por la economía es una agregación CES de $r$ variedades de estos, dada por la siguiente ecuación:

$$
P_{i m, t}=\left[\int_{0}^{1} P(r)^{1-e_{i m}} d r\right]^{\frac{1}{1-e_{i m}}}, e_{i m}>1 \quad \text { y } \quad \forall r .
$$

\section{- Precios agregados}

El índice de precios total de la economía es una agregación CES, tanto del índice de precios del bien nacional como del bien importado.

$$
P_{T, t}=\left[(1-\alpha)\left(P_{d, t}\right)^{1-e}+\alpha\left(\epsilon_{t} P_{t}^{*}\right)^{1-e}\right]^{\frac{1}{1-e}} .
$$

Además, como $S_{t}=\frac{\epsilon_{t} P_{t}^{*}}{P_{d, t}}$, se tendrá que:

$$
\frac{P_{T, t}}{P_{d, t}}=\left[(1-\alpha)+\alpha\left(S_{t}\right)^{1-e}\right]^{\frac{1}{1-e}} .
$$

Linealizando esta expresión de manera análoga a la estructura del consumo agregado a través de una aproximación de Taylor se tiene:

$$
\hat{p}_{T, t}-\hat{p}_{d, t}=\alpha \hat{s}_{t}
$$


En ese sentido, linealizando los resultados, la ecuación de consumo doméstico es: $\hat{c}_{d, t}=e \alpha \hat{s}_{t}+\hat{c}_{T, t}$. El consumo de bienes importados es: $\hat{c}_{i m, t}=-e(1-\alpha) \hat{s}_{t}+\hat{c}_{T, t}$.

\section{D. Términos de intercambio}

Los términos de intercambio bilaterales entre la economía doméstica y el país $r$ se definen como:

$$
S_{t}=\left[\int_{0}^{1} S_{t}(r)^{1-e_{i m}} d r\right]^{\frac{1}{1-e_{i m}}}, e_{i m}>1 \quad \text { y } \quad \forall r .
$$

Los términos de intercambio efectivos son el nivel de precios en el extranjero, expresado en moneda nacional, y dividido por el precio de bienes domésticos. Los términos de intercambio se deben incluir al momento de modelar el comportamiento de una economía abierta, aunque no se van a considerar shocks de los mismos. Por tanto, la ecuación simplificada es:

$$
S_{t}=\frac{P_{i m, t}}{P_{d, t}}
$$

linealizando esta última expresión alrededor de un estado de equilibrio simétrico satisfactorio. Este estado se logra asumiendo que $e_{i m}=0$, con lo cual $S=\int_{0}^{1} S(r) d r=1$. De manera similar, los demás estados estacionarios se definirán de esta forma:

$$
\hat{s}_{t}=\hat{p}_{i m, t}-\hat{p}_{d, t},
$$

y de esta manera se tiene que:

$$
\hat{s}_{t}=\int_{0}^{1} \hat{s}_{t}(r) d r, \quad \forall r .
$$

\section{E. Tipo de cambio real}

Se define como la relación entre el poder de compra de una divisa en relación con otra divisa. El poder de compra de una divisa es la cantidad de 
Gil-León y Suárez-Cante: Implicaciones de los choques de prima de riesgo en una economía...

bienes que pueden ser comprados con una unidad de esa divisa. El poder de compra de la moneda en un país depende del nivel de precios. El poder de compra de una moneda en el extranjero depende de la tasa de cambio nominal y de los precios en el extranjero.

Entonces, el tipo de cambio real pone de manifiesto que la dinámica inflacionaria en pequeñas economías abiertas difiere de la dinámica inflacionaria en economías cerradas, debido a los diferenciales de precios entre los bienes producidos domésticamente y los producidos en el extranjero. Como $S_{t}$ es el tipo de cambio nominal, entonces se define el tipo de cambio real en forma linealizada como:

$$
\widehat{t c r}_{t}=\widehat{\varepsilon}_{t}+\hat{p}_{t}^{*}-\hat{p}_{T, t} .
$$

Desagregando la definición:

$$
\widehat{t c r}_{t}=\hat{s}_{t}(1-\alpha)=\hat{s}_{t}-\hat{p}_{t}^{*}+\hat{p}_{d, t}+\hat{p}_{t}^{*}-\hat{p}_{T, t}
$$

\section{Anexo 2. Shock tecnológico}

Figura 2A. Transmisión del shock tecnológico sobre las variables
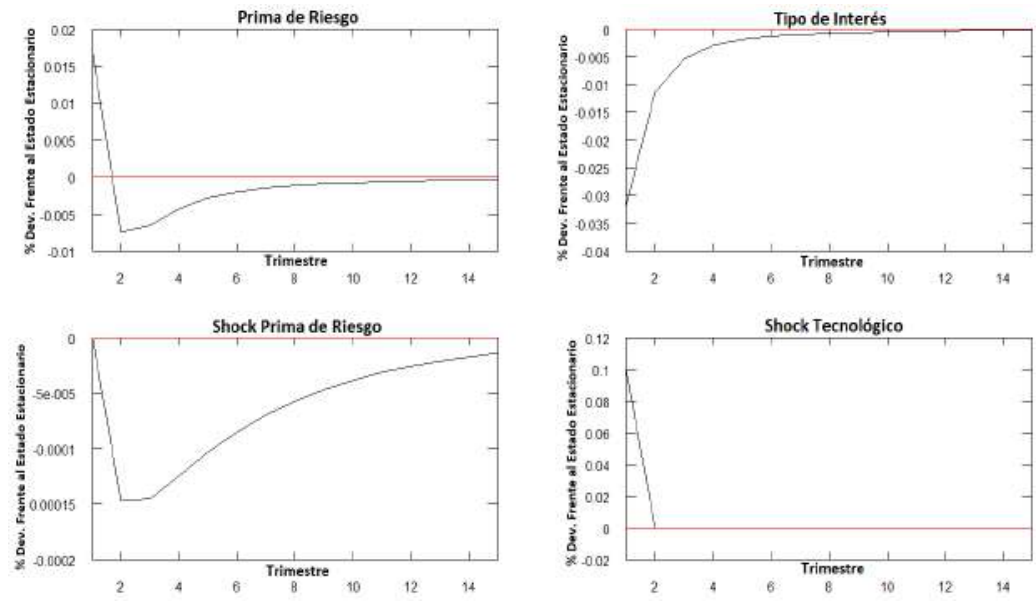

Fuente: elaboración propia. 


\section{Referencias}

Alendal, L. A. (2008). Estimating the New Keynesian Phillips Curve in an Open Economy DSGE Framework (Tesis de maestría, University of Oslo, Noruega). Recupeado de: https://www.semanticscholar.org/paper/Esti mating-the-New-Keynesian-Phillips-Curve-in-an-Alendal/4e019111 88cd23ecbe25a3a61798c627e438a3b5

Amano, R. \& Shukayev, M. (2012). Risk Premium Shocks and the Zero Bound on Nominal Interest Rates. Journal of Money, Credit and Banking, 44(8), 1475-1505.

Bergholt, D. (2015). Foreign Shocks In An Estimated Multi-Sector Model (CAMP Working Paper Series No. 4/2014). Recuperado del sitio web de BI Norwegian Business School: https:/www.bi.edu/globalassets/fors kning/camp/working-papers/2014/working_camp_4-2014.pdf

Bonaldi, D., González, A. \& Rodríguez, D. (2010). Importancia de las rigideces reales y nominales en Colombia: un enfoque de equilibrio general dinámico y estocástico (Borradores de Economía, No. 591). Recuperado del sitio web del Banco de la Republica: https://www.banrep.gov.co/sites/default/file s/publicaciones/pdfs/borra591.pdf

Eraker, B. \& Wang, J. (2015). A Non-Linear Dynamic Model of the Variance Risk Premium. Journal of Econometrics, 187(2), 547-556: https://www. sciencedirect.com/science/article/abs/pii/S0304407615000639

Galí, J. (2008). Monetary Policy, Inflation, and the Business Cycle. New Jersey: Princeton University Press.

Gelain, P. (2010). The External Finance Premium in the Euro: Area a Useful Indicator for Monetary Policy? (European Central Bank, No. 1171). Recupeado del sitio web del European Central Bank: https://www.ec b.europa.eu/pub/pdf/scpwps/ecbwp1171.pdf?cce211b02fac850a7aa0c d6b55fccae0

Gil, J. M. (2015). Relación, Estabilidad Financiera y Política Monetaria: Análisis para Colombia. Ensayos sobre Politica Económica, 33(77), 133-148. 
Gil-León y Suárez-Cante: Implicaciones de los choques de prima de riesgo en una economía...

González, A., Mahadeva, L., Prada, J. D. \& Rodríguez, D. (2011). Policy analysis tool applied to Colombian needs: PATACON model description. Ensayos sobre Politica Económica, 29(66), 222-245.

González, A., López, M., Rodríguez, N. \& Téllez, S. (2014). Fiscal policy in a small open economy with oil sector and non-ricardian agents. Desarrollo y Sociedad, 73, 33-69.

Graeve, F. D., Dossche, M., Emiris, M., Sneessens, H. \& Wouters, R. (2010). Risk premiums and macroeconomic dynamics in a heterogeneous agent model. Journal of Economic Dynamics \& Control, 34(9), 1680-1699: ht tps://www.sciencedirect.com/science/article/pii/S0165188910001557

Hernández Henao, J. M. (2013). Demanda externa, términos de intercambio y el papel de la política monetaria durante la crisis de 2008 (Documentos de Investigación No. 7). Recuperado del Centro de Estudios Monetarios Latinomaericanos: https://www.cemla.org/PDF/investigacion/inv-2013 -02-07.pdf

Iranzo, S. (2008). Introducción Al riesgo-pais. Madrid: Banco de España.

López, E. \& Ramírez, M. T. (2011). Formación de salarios y precios en Colombia. Bogotá, D. C.: Banco de la República.

López, M., Prada, J. \& Rodríguez, N. (2008). Small Open Economy and Financial Accelerator Mechanism (Borradores de Economía, No. 35). Recuperado del Banco de la República: http://repositorio.banrep.g ov.co/bitstream/handle/20.500.12134/5542/be_525.pdf?sequence = $1 \&$ isAllowed $=\mathrm{y}$

Melo, L. \& Riascos, A. (2004). Sobre los efectos de la politica monetaria en Colombia. Recuperado de: http://www.banrep.org/docum/ftp/borra28 1.pdf

Mickelsson, G. (2009). Monetary Policy in Closed and Open Economies (Tesis de maestría, Uppsala Universitet, Suecia). Recuperado de: http://www. diva-portal.org/smash/get/diva2:233883/FULLTEXT01.pdf 
Papantonis, I. (2016). Volatility risk premium implications of GARCH option pricing models. Economic Modelling, 58, 104-115.

Pienika, E. (2014). Un modelo de equilibrio general dinámico y estocástico con rigideces nominales para la economía uruguaya (Tesis de maestría, Universidad Torcuato Di Tella, Argentina). Recupeardo de: https://re positorio.utdt.edu/handle/utdt/1894

Posch, O. (2009). Risk premia in general equilibrium (CREATES Research Papers No. 2009-58). Recuperado del sitio web de Aarhus University: https:/www.econ.au.dk/fileadmin/site_files/filer_oekonomi/Workin g_Papers/CREATES/2009/rp09_58.pdf

Ramirez, F., \& Torres, F. (2013). A stochastic and dynamic general equilibrium model with nominal rigidities for policy analysis and forecast in the Dominican Republic. Munich Personal RePEc Archive, 53.

Rudebusch, G. D. \& Swanson, E. T. (2008). The Bond Premium in a DSGE Model with Long-Run Real and Nominal Risks (Federal Reserve Bank of San Francisco No. 42). Recuperado del sitio web de SSRN's eLibrary: https://papers.ssrn.com/sol3/papers.cfm?abstract_id=1364348

Rudolf, B. \& Zurlinden, M. (2014). A compact open economy DSGE model or Switzerland (SNB Economic Studies, 2014-08). Recuperado del sitio web del Swiss National Bank: snb.ch/n/mmr/reference/economic_studi es_2014_08/source/economic_studies_2014_08.n.pdf

Santana, J. C. (2016). La estimación de un modelo hibrido DSGEVAR(LAMBDA): Una Aplicación para Colombia (Tesis de maestría, Universidad Nacional de Colombia). Recuperado de: http://bdigital.u nal.edu.co/54689/1/79986837.2016.pdf

Soares , C. C., Portugal, M. S. \& Bejarano Aragón, E. K. (2016). Assessing Brazilian macroeconomic dynamics using a Markov-switching DSGE model. Economia, 17(1), 23-42.

Suescún, R. \& Steiner, R. (2017). Un Modelo De Equilibrio General DInámico Para La Evauación De La Política Económica De Colombia. Recuperado de: https://www.repository.fedesarrollo.org.co/handle/11445/3449 
Gil-León y Suárez-Cante: Implicaciones de los choques de prima de riesgo en una economía...

Vallejos, M. \& Navia, S. (2016). Perturbaciones externas e internas para una economía pequeña y abierta: un anális microfundado para el caso boliviano: periodo 2000-2015. La paz: Ministerio de Economía y Finanzas Públicas.

Woodford, M. (2003). Interest and Price: Foundations of a Theory of Monetary Policy. New Jersey: Princeton University Press. 